\title{
Non-covariance of the dressed-metric approach in loop quantum cosmology
}

\author{
Martin Bojowald* \\ Institute for Gravitation and the Cosmos, \\ The Pennsylvania State University, \\ 104 Davey Lab, University Park, PA 16802, USA
}

\begin{abstract}
The dressed-metric approach is shown to violate general covariance by demonstrating that it cannot have an off-shell completion in which the correct infinitesimal relations of space-time hypersurface deformations are realized. The main underlying reason - a separation of background degrees of freedom and modes of inhomogeneity that is incompatible with covariance - is shared with other approaches such as hybrid loop quantum cosmology.
\end{abstract}

\section{Introduction}

The dressed-metric approach [1] is an attempt to extend modified Friedmann equations of loop quantum cosmology to perturbative inhomogeneity in order to describe structure formation. If any such proposal is to be consistent, it must respect general covariance in some form to guarantee that the equations are meaningful: If general covariance is violated, the theory is either plagued by spurious, unphysical degrees of freedom if one decides to impose a restriced number of covariance transformations (or none at all), or it is overconstrained if broken covariance transformations are imposed which then identify physical solutions that are supposed to be distinct. A non-covariant modification of a covariant theory has either too many or too few propagating degrees of freedom, depending on how it is applied.

Since loop quantum cosmology [2] modifies the background dynamics of a homogeneous universe, perturbative inhomogeneity is not guaranteed to obey covariance conditions. However, the dressed-metric approach assumes that classical observables and Hamiltonians can be used for inhomogeneity without modifications even while the background dynamics is modified such that it may allow a bounce, a crucial ingredient in some of the developed scenarios. In this paper, we provide the first analysis of covariance in the dressed-metric approach, pointing out several previously overlooked subtleties and ultimately reaching the conclusion that covariance is violated.

Several details of the technical implementation of the dressed-metric approach obscure the issue of covariance, which is perhaps the reason why this important issue has not been addressed yet. The approach postulates separate quantizations for an isotropic background

\footnotetext{
*e-mail address: bojowald@gravity.psu.edu
} 
space-time and inhomogeneous perturbations on it, even though the degrees of freedom of both ingredients are interrelated in any covariant setting that obtains background and perturbations from an expansion of a covariant theory. For instance, the limited covariance transformations that remain in a spatially homogeneous reduction of a covariant theory do not restrict the possible dynamics, which can be modified at will. Homogeneous background dynamics that can be obtained from some higher-curvature action, by contrast, is not arbitrary but subject to conditions that implicitly ensure its descendance from a covariant theory of this type. Once a covariant theory has been restricted to homogeneity, however, the dynamics can be modified consistently in the homogeneous setting, without any restrictions that would result from covariance or integrability conditions in an inhomogeneous theory. By separating the degrees of freedom into background and perturbations before implementing quantum modifications, and then leaving the perturbative degrees of freedom unmodified, the dressed-metric approach construes a setting in which the usual covariance conditions are relaxed. This observation does not directly imply that the approach violates covariance, but it shows that any analysis of covariance in this approach is subtle and must be performed in detail.

While covariance itself has not yet been analyzed in the dressed-metric approach, some transformations related to this condition have been discussed in the seminal papers. However, these transformations, like the implementation of degrees of freedom, act separately on background and perturbations and do not respect the interrelated nature of these degrees of freedom with respect to covariance. In particular, the dressed-metric approach replaces linear perturbations of metric and extrinsic curvature, or of other fields used in canonical gravity, by Bardeen potentials or curvature perturbations [3, 4]. Since these variables are invariant with respect to small inhomogeneous coordinate transformations, they respect some partial form of covariance. The homogeneous background dynamics, meanwhile, is made invariant with respect to homogeneous time reparameterizations by using the method of deparameterization [5, 6], formulating homogeneous evolution not with respect to a time coordinate but rather with respect to one of the dynamical fields of the theory, given by a free massless scalar. The resulting framework is formally consistent because no time coordinate is used explicitly, and spatial coordinates can be adapted to the homogeneous background. In this sense, the dressed-metric approach constructs a consistent quantum-field theory on a modified homogeneous space-time, but it does not show that fields and background can be part of a common covariant theory. Therefore, it is not clear whether it can rightfully be considered a description of cosmological evolution in quantum gravity, or of quantum space-time.

The treatment of transformations in the dressed-metric approach suffers from several old and new problems:

- While deparameterization eliminates the appearance of coordinate time, as applied in [1] it selects a specific reference scalar field as internal time (which has to be free and massless in order to play the role of a global measure of time). In models in which more than one choice of global internal time are available, quantum corrections in general imply inequivalent observables depending on which internal time is used 
[7, 8, 9]. Even if one does not refer to coordinate time, therefore, time reparameterization invariance is not guaranteed after quantization. This problem, which is being investigated with several methods, see for instance [10, 11, 12, 7, 8, 13, 14, 15, 16], is not specific to the dressed-metric approach and will therefore be disregarded here.

- Bardeen potentials, in spite of one of their common names, are not gauge invariant [17. 1. They are invariant with respect to small inhomogeneous coordinate transformations in a perturbative setting, but they are no longer invariant if one or both of the two implied conditions, smallness and inhomogeneity, is violated. Curvature perturbations, which are available in the presence of a scalar matter field, are invariant provided only that coordinate transformations are small and not necessarily inhomogeneous, but even this condition is not met by all transformations relevant for perturbative cosmology: While a first-order description of inhomogeneity need not consider higher than first-order transformations, it should include large homogeneous coordinate changes such as a transformation from proper time to conformal time. In the dressed-metric approach, homogeneous coordinate transformations are implemented by deparameterization for the background, separately from the inhomogeneous sector even though they act non-trivially on Bardeen potentials and curvature perturbations when they are large.

- A detailed analysis of space-time transformation in a 4-dimensional or a canonical setting, presented in the next section, shows that background transformations and those acting on perturbations do not form a direct but rather a semidirect product. This important algebraic structure is violated by the separation of background and perturbation degrees of freedom imposed by the dressed-metric approach, which would be compatible only with a direct product. As a consequence, by its very construction the dressed-metric approach is unable to provide the correct off-shell structure required for a covariant theory of background and perturbations.

\section{Space-time structure}

The perturbative form of covariance is somewhat different depending on whether one uses a formulation of tensor fields in four dimensions or a canonical description. However, both viewpoints lead to the same conclusion that background and perturbative transformations form a semidirect product.

\subsection{4-dimensional formulation}

Background coordinate transformations affect only time $t$ and are generated by vector fields of the form $f(t) \partial / \partial t$ with an arbitrary function $f(t)$. Perturbative coordinate changes are

\footnotetext{
${ }^{1}$ The relevance of this fact for the dressed-metric approach has been pointed out privately by Robert Brandenberger and Neil Turok.
} 
generated by vector fields $\xi^{\alpha} \partial / \partial x^{\alpha}$ with four components $\xi^{\alpha}$ which are small in the sense that any products of multiple $\xi^{\alpha}$ or of $\xi^{\alpha}$ with perturbative fields are ignored. Bardeen potentials and curvature perturbations are constructed by ensuring $\xi^{\alpha}$-independence of suitable combinations of metric components, but they do not consider $f(t)$ (unless this function is small and may be considered a contribution to $\left.\xi^{0}\right)$.

\subsubsection{Bardeen potentials and curvature perturbations}

Specifically, we may transform metric components by inserting small coordinate changes $x^{\alpha} \mapsto x^{\alpha}+\xi^{\alpha}$ in the line element

$$
\mathrm{d} s^{2}=a^{2}\left(-(1+2 \phi) \mathrm{d} \eta^{2}+2 \partial_{i} B \mathrm{~d} \eta \mathrm{d} x^{i}+\left((1-2 \psi) \delta_{i j}+2\left(\partial_{i} \partial_{j}-\frac{1}{3} \delta_{i j} \Delta\right) E\right) \mathrm{d} x^{i} \mathrm{~d} x^{j}\right)
$$

for linear scalar perturbations on a flat isotropic background, here using conformal time $\eta$ and including only scalar modes. We distinguish between time transformations, $\eta \mapsto \eta+\xi^{0}$, and scalar spatial transformations, $x^{i} \mapsto x^{i}+\partial^{i} \xi$ with a scalar function $\xi$. In the first case, denoting a derivative with respect to $\eta$ by a prime,

$$
\mathrm{d} \eta^{2} \mapsto \mathrm{d} \eta^{2}+2 \xi^{0 \prime} \mathrm{d} \eta^{2}+2 \partial_{i} \xi^{0} \mathrm{~d} \eta \mathrm{d} x^{i}
$$

to first order in $\xi^{0}$, while $a(\eta)^{2} \mapsto a(\eta)^{2}\left(1+2 a^{\prime} \xi^{0} / a\right)$. Rearranging the resulting line element to bring it back to the old form (11) but with adjusted scalar perturbations, we obtain the transformations

$$
\phi \mapsto \phi+\xi^{0 \prime}+\frac{a^{\prime}}{a} \xi^{0} \quad, \quad \psi \mapsto \psi-\frac{a^{\prime}}{a} \xi^{0} \quad, \quad B \mapsto B-\xi^{0} \quad, \quad E \mapsto E .
$$

Notice that the transformation of $B$ follows only if $\partial_{i} \xi^{0} \neq 0$ in (2) because the line element depends on $\partial_{i} B$ but not directly on $B$. Therefore, for spatially constant $\xi^{0}$, or a small background transformation, there is no need for $B$ to change, in contrast to (31). In fact, the transformation of $B$ is undetermined in this case because $B \mapsto B-\alpha \xi^{0}$ would be consistent for any real $\alpha$. This ambiguity is not relevant in the line element, which only depends on $\partial_{i} B$, but it implies an ambiguity in the Bardeen potentials, which depend directly on $B$ and not just its spatial derivatives. Thus we obtain a distinction between background and perturbation transformations even if both are small.

For small spatial transformations, we insert

$$
\delta_{i j} \mathrm{~d} x^{i} \mathrm{~d} x^{j} \mapsto \delta_{i j} \mathrm{~d} x^{i} \mathrm{~d} x^{j}+2 \partial_{i} \xi^{\prime} \mathrm{d} \eta \mathrm{d} x^{i}+2 \partial_{i} \partial_{j} \xi \mathrm{d} x^{i} \mathrm{~d} x^{j}
$$

in the line element and read off

$$
\phi \mapsto \phi \quad, \quad \psi \mapsto \psi \quad, \quad B \mapsto B+\xi^{\prime} \quad, \quad E \mapsto E+\xi
$$

Therefore, $\phi, \psi$ and $B-E^{\prime}$ are invariant with respect to spatial transformations. (Again, the transformation of $B$ would be undetermined if $\partial_{i} \xi=0$, but for spatial transformations 
we need $\partial_{i} \xi \neq 0$ in order to have a non-trivial $\xi_{i}=\partial_{i} \xi \neq 0$.) Since $B-E^{\prime}$ changes to $B-E^{\prime}-\xi^{0}$ by a time transformation, the combinations

$$
\Phi:=\phi+\frac{a^{\prime}}{a}\left(B-E^{\prime}\right)+\left(B-E^{\prime}\right)^{\prime} \quad \text { and } \quad \Psi:=\psi-\frac{a^{\prime}}{a}\left(B-E^{\prime}\right)
$$

are invariant, provided $\xi^{0}$ is not spatially constant.

If there is a matter scalar field, $\varphi=\bar{\varphi}+\delta \varphi$, its perturbation transforms by $\delta \varphi \mapsto \delta \varphi+$ $\bar{\varphi}^{\prime} \xi^{0}$. Therefore, one can obtain $\xi^{0}$-independent combinations, the curvature perturbations

$$
\begin{aligned}
& \mathcal{R}_{1}=\psi+\frac{a^{\prime}}{a \bar{\varphi}^{\prime}} \delta \varphi \\
& \mathcal{R}_{2}=\phi-\frac{1}{2}\left(\frac{a}{a^{\prime}}\right)^{\prime} \psi-\frac{1}{\bar{\varphi}^{\prime}}\left(\frac{a^{\prime}}{a}-\frac{\bar{\varphi}^{\prime \prime}}{\bar{\varphi}^{\prime}}\right) \delta \varphi+\frac{1}{2} \frac{a}{a^{\prime}} \psi^{\prime}-\frac{1}{2 \bar{\varphi}^{\prime}} \delta \varphi^{\prime}
\end{aligned}
$$

without using $B$. These perturbations, unlike Bardeen potentials, are invariant also with respect to spatially constant $\xi^{0}$, but not with respect to large background transformations.

Formulating the dressed-metric approach using curvature perturbations instead of Bardeen potentials implies that we do not have to distinguish between small background transformations and perturbative transformations. However, there remain non-trivial large background transformations, hence the additional step of deparameterization in the approach. Large background transformations change curvature perturbations merely by reparameterizations, such as replacing $a^{\prime} /\left(a \bar{\varphi}^{\prime}\right)$ with $\dot{a} / \dot{\bar{\varphi}}$ when transforming from conformal time to proper time. Formally, the approach therefore does take into account all relevant transformations. However, the way it does so violates the required off-shell structure of background and perturbative transformations.

\subsubsection{Algebraic structure}

Background and perturbative transformations are not independent but algebraically related. The commutator of two such transformations or of their generating vector fields, given by

$$
\left[f(t) \frac{\partial}{\partial t}, \xi^{\alpha} \frac{\partial}{\partial x^{\alpha}}\right]=f \dot{\xi}^{\alpha} \frac{\partial}{\partial x^{\alpha}}-\dot{f} \xi^{0} \frac{\partial}{\partial t},
$$

is a perturbative transformation. Using pairs

$$
\left(f, \xi^{\alpha}\right) \in \mathcal{V}_{\text {background }} \oplus \mathcal{V}_{\text {pert }}=\mathcal{V}
$$

of background and perturbation vector fields, arranged by perturbative order to make the algebraic structure more clear, the combination of both types of transformations is therefore a semidirect product:

$$
\left[\left(f_{1}, \xi_{1}^{\alpha}\right),\left(f_{2}, \xi_{2}^{\alpha}\right)\right]=\left(f_{1} \dot{f}_{2}-f_{2} \dot{f}_{1}, \zeta^{\alpha}\right)
$$

with

$$
\zeta^{\alpha}=f_{1} \dot{\xi}_{2}^{\alpha}-f_{2} \dot{\xi}_{1}^{\alpha}-\delta_{0}^{\alpha}\left(\dot{f}_{1} \xi_{2}^{0}-\dot{f}_{2} \xi_{1}^{0}\right)
$$


depending on $\xi_{1}$ and $\xi_{2}$ as well as $f_{1}$ and $f_{2}$.

This bracket shows that $\mathcal{V}_{\text {background }}$ is non-Abelian, with bracket $\left[f_{1}, f_{2}\right]_{\text {background }}=$ $f_{1} \dot{f}_{2}-f_{2} \dot{f}_{1}$, while $\mathcal{V}_{\text {pert }}$ is Abelian, $\left[\xi_{1}^{\alpha}, \xi_{2}^{\alpha}\right]_{\text {pert }}=0$. However, the full bracket in $\mathcal{V}$ has an extra term $\zeta^{\alpha}$, which can be written as $\zeta^{\alpha}=\phi\left(f_{1}\right) \xi_{2}^{\alpha}-\phi\left(f_{2}\right) \xi_{1}^{\alpha}$ with the homomorphism

$$
\phi(f) \xi^{\alpha}=f \dot{\xi}^{\alpha}-\delta_{0}^{\alpha} \dot{f} \xi^{0}
$$

from $\mathcal{V}_{\text {background }}$ to the derivations on $\mathcal{V}_{\text {pert }}$. (It clearly maps to derivations because $\mathcal{V}_{\text {pert }}$ is Abelian. The homomorphism property can be shown by a direct calculation.) Therefore, the bracket on $\mathcal{V}$ can be written as

$$
\left[\left(f_{1}, \xi_{1}^{\alpha}\right),\left(f_{2}, \xi_{2}^{\alpha}\right)\right]=\left(\left[f_{1}, f_{2}\right]_{\text {background }},\left[\xi_{1}^{\alpha}, \xi_{2}^{\alpha}\right]_{\text {pert }}+\phi\left(f_{1}\right) \xi_{2}^{\alpha}-\phi\left(f_{2}\right) \xi_{1}^{\alpha}\right)
$$

identifying

$$
\mathcal{V}=\mathcal{V}_{\text {background }} \ltimes_{\phi} \mathcal{V}_{\text {pert }}
$$

as the semidirect product of the Lie algebras $\mathcal{V}_{\text {background }}$ and $\mathcal{V}_{\text {pert }}$.

We do not have a direct product that could be implemented by separate treatments of invariance, such as deparameterization for the background and curvature perturbations for the inhomogeneous fields. While the dressed-metric approach is formally consistent in that it eliminates the relevant transformations, it does so incorrectly by ignoring their interrelated off-shell nature. In the next section we will demonstrate explicitly that there is no off-shell completion of the attempted invariance proposed by the dressed-metric approach, but first we review the off-shell structure in a canonical setting.

\subsection{Canonical formulation}

One might think that the canonical formulation should not have a non-zero commutator of background and perturbative transformations because fields on a fixed spatial slice do not have any time dependence, such that the time derivatives on the right-hand side of (8) vanish. (Time dependence in canonical transformations is not explicit but implemented by an additional term added to the usual constraints which depends on momenta of lapse and shift and has coefficients given by initial values of time derivatives of the fields [18, 19].) However, the canonical description must be equivalent to the 4-dimensional formulation, and therefore should give rise to a related semidirect product of background and perturbative transformations. The main mathematical difference is that canonical transformations form a Lie algebroid [20, 21] rather than a Lie algebra.

\subsubsection{Algebroid}

Geometrically, the product remains semidirect, not because of time derivatives but because the canonical generators, given by constraints, refer to directions normal to spatial slices rather than time directions determined by a coordinate [22]. As a consequence, a perturbative inhomogeneous transformation changes the normal directions, such that a subsequent 


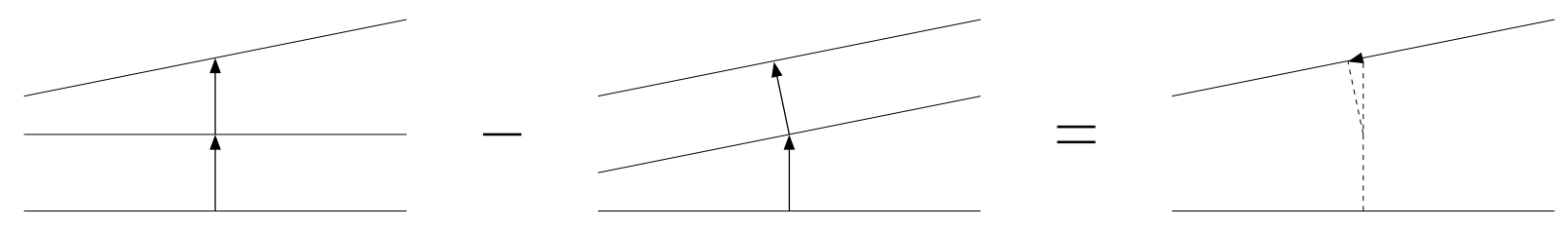

Figure 1: Non-zero commutator of a homogeneous background transformation and a perturbative transformation (here, linear spatial dependence), equal to a non-zero spatial displacement.

background transformation acquires new directions compared with one applied before the inhomogeneous transformation; see Fig. 1.

The specific commutator follows from a restriction of the full hypersurface-deformation brackets of the Hamiltonian and diffeomorphism constraints, $H[N]$ and $D\left[M^{a}\right]$. We obtain background transformations by applying the Hamiltonian constraint to homogeneous lapse functions $\bar{N}$, while perturbative inhomogeneous constraints are obtained by specializing the Hamiltonian constraint to a small inhomogeneous perturbation, $\delta N$, and the diffeomorphism constraint to a small inhomogeneous vector field, $\delta M^{a}$. The leading perturbative expressions are then obtained by expanding the constraints $H[\delta N]$ and $D\left[\delta M^{a}\right]$ up to quadratic dependence on the fields, counting $\delta N$ and $\delta M^{a}$ as first-order contributions. The general bracket [23]

$$
\left[H\left[N_{1}\right], H\left[N_{2}\right]\right]=D\left[q^{a b}\left(N_{1} \partial_{b} N_{2}-N_{2} \partial_{b} N_{1}\right)\right]
$$

with the inverse spatial metric $q^{a b}=a^{-2} \delta^{a b}$ then turns into

$$
[H[\bar{N}], H[\delta N]]=D\left[a^{-2} \bar{N} \partial^{a} \delta N\right]
$$

with a non-zero right-hand side. More generally,

$$
\left[H\left[\bar{N}_{1}+\delta N_{1}\right], H\left[\bar{N}_{2}+\delta N_{2}\right]\right]=D\left[a^{-2}\left(\bar{N}_{1} \partial^{a} \delta N_{2}-\bar{N}_{2} \partial^{a} \delta N_{1}\right)\right]
$$

while all brackets involving $D\left[\delta M^{a}\right]$ are zero to first perturbative order.

In the canonical formulation, the background bracket is Abelian because $\left[H\left[\bar{N}_{1}\right], H\left[\bar{N}_{2}\right]\right]=$ 0 for any spatially constant $\bar{N}_{1}$ and $\bar{N}_{2}$. The generators of perturbative inhomogeneous transformations, $H[\delta N]$ and $D\left[\delta M^{a}\right]$, also form an Abelian Lie algebra, because the righthand side of (15) vanishes to the order considered here when both $N_{1}=\delta N_{1}$ and $N_{2}=\delta N_{2}$ are of first order. The Lie-algebroid structure of the full bracket (15) therefore seems to remain only in the non-trivial relation (16) between background and perturbation generators. It is nevertheless possible to interpret both background and perturbations as Lie algebroids, $\mathcal{E}_{\text {background }}$ and $\mathcal{E}_{\text {pert }}$, respectively, over the same base manifold $X_{\text {pert }}$ of perturbed metrics. (Background metrics might seem sufficient for $\mathcal{E}_{\text {background }}$, but using the same base manifold for $\mathcal{E}_{\text {background }}$ and $\mathcal{E}_{\text {pert }}$ is convenient for the construction of a semidirect product.)

With a base manifold of metrics, (17) determines the algebroid bracket only for constant sections, that is $\bar{N}$ and $\left(\delta N, \delta M^{a}\right)$ which do not depend on the metric (while the 
perturbations $\delta N$ and $\delta M^{a}$ may always depend on the spatial position). If we allow metric-dependent functions, the Lie algebroid $\mathcal{E}_{\text {background }}$ is no longer Abelian because the background part of the bracket (17) should then be generalized to

$$
\left[H\left[\bar{N}_{1}\right], H\left[\bar{N}_{2}\right]\right]=H\left[\bar{N}_{1} \delta_{n} \bar{N}_{2}-\bar{N}_{2} \delta_{n} \bar{N}_{1}\right]
$$

where $\delta_{n} N=\left(\delta N / \delta q_{a b}\right) \mathcal{L}_{n} q_{a b}$ is the normal derivative of $N$, constructed by the chain rule using the Lie derivative $\mathcal{L}_{n}$ along the vector field normal to hypersurfaces. (This extension can be derived from the Poisson bracket of Hamiltonian constraints with metric-dependent lapse functions.) The Lie algebroid $\mathcal{E}_{\text {pert }}$ remains Abelian because the right-hand side of an equation analogous to (18) with $\bar{N}$ replaced by $\delta N$ would be of second order.

The anchor map of a Lie algebroid $\mathcal{E}$, defined as

$$
\rho: \Gamma(T \mathcal{E}) \rightarrow \Gamma(T X)
$$

such that

$$
\left[e_{1}, f e_{2}\right]=f\left[e_{1}, e_{2}\right]+\left(\rho\left(e_{1}\right) f\right) e_{2}
$$

for any $e_{1}, e_{2} \in \Gamma(T \mathcal{E})$ and $f \in C^{1}(X)$, is necessarily zero for Abelian brackets, that is for $\mathcal{E}_{\text {pert }}$ in our case. The non-Abelian bracket of $\mathcal{E}_{\text {background }}$ is compatible with the anchor map $\bar{N} \mapsto \delta q_{a b}=\bar{N} \mathcal{L}_{n} q_{a b}$. These two anchor maps are equivalent to the first-order perturbative content of the full anchor, given by $\left(N, M^{a}\right) \mapsto \delta q_{a b}=\mathcal{L}_{N n+M} q_{a b}[20$.

Abstractly, we denote elements in the fiber of the first Lie algebroid, $\mathcal{E}_{\text {background }}$, simply by $\bar{N} \in \mathbb{R}$. Elements of fiber of the second Lie algebroid, $\mathcal{E}_{\text {pert }}$ which is Abelian, are given by $\left(\delta N, \delta M^{a}\right)$, where $\delta N$ and $\delta M^{a}$ depend on the spatial position and therefore form infinite-dimensional fibers. The map

$$
\psi(\bar{N})\left(\delta N, \delta M^{a}\right)=\left(0, a^{-2} \bar{N} \partial^{a} \delta N\right)
$$

defines a Lie algebroid morphism from $\mathcal{E}_{\text {background }}$ to the derivations on $\mathcal{E}_{\text {pert }}$. (This map is well-defined because background metrics, parameterized by the scale factor $a$, are included in both base manifolds. It maps to derivations because $\mathcal{E}_{\text {pert }}$ is Abelian. In order to show the morphism property, note that $\delta_{n} \bar{N}$ is of first order, such that $\delta_{n} \bar{N} \partial^{a} \delta N \sim 0$ is of second order and therefore treated as zero.)

The bracket (17) together with the vanishing brackets involving spatial deformations now appear in the form

$$
\left[\left(\bar{N}_{1},\left(\delta N_{1}, \delta M_{1}^{a}\right)\right),\left(\bar{N}_{2},\left(\delta N_{2}, \delta M_{2}^{a}\right)\right)\right]=\left(\left[\bar{N}_{1}, \bar{N}_{2}\right], \psi\left(\bar{N}_{1}\right)\left(\delta N_{2}, \delta M_{2}^{a}\right)-\psi\left(\bar{N}_{2}\right)\left(\delta N_{1}, \delta M_{1}^{a}\right)\right),
$$

which is the same as the bracket of a semidirect products of Lie algebroids defined in [24], where the analog of $\mathcal{E}_{\text {pert }}$ (but not of $\mathcal{E}_{\text {background }}$ ) is required to be Abelian in to avoid obstructions. The general construction determines a semidirect product with anchor map inherited directly from $\mathcal{E}_{\text {background }}$, just as we have found here. We therefore have shown that

$$
\mathcal{E}=\mathcal{E}_{\text {background }} \ltimes_{\psi} \mathcal{E}_{\text {pert }} .
$$


Comparing with the 4-dimensional perspective, although the precise algebraic structure of canonical transformations is rather different from that found in Section 2.1.2, the bracket of a semidirect product of background and perturbation transformations is obtained in both cases.

\subsubsection{Poisson structure}

A formal derivation of the crucial equation (16) through Poisson brackets of phase-space representations of the hypersurface-deformation generators shows the interplay of different perturbative orders in this result. Following the formalism developed in [25, 26], or [27] for canonical perturbation theory in metric variables, we coordinatize the gravitational phase space in triad form, given by the components $E_{i}^{a}$ of a densitized triad and the corresponding components of extrinsic curvature, $K_{a}^{i}$. With perturbative inhomogeneity, we write $E_{i}^{a}=p \delta_{i}^{a}+\delta E_{i}^{a}$ and $K_{a}^{i}=k \delta_{a}^{i}+\delta K_{a}^{i}$, where the background variables $p$ and $k$ depend only on time and their internal frame has been fixed by choosing the background fields to be proportional to the Kronecker delta. For simplicity, we will assume that $p>0$, fixing the orientation of space. The background variables can then be derived from the fields by integrating over a fixed spatial region $\mathcal{V}$ of coordinate volume $V_{0}=\int_{\mathcal{V}} \mathrm{d}^{3} x$ :

$$
p=\frac{1}{V_{0}} \int_{\mathcal{V}} E_{i}^{a} \delta_{a}^{i} \mathrm{~d}^{3} x \quad, \quad k=\frac{1}{V_{0}} \int_{\mathcal{V}} K_{a}^{i} \delta_{i}^{a} \mathrm{~d}^{3} x .
$$

In order to avoid double-counting the background variables, we impose linear second-class constraints

$$
\int_{\mathcal{V}} \delta E_{i}^{a} \delta_{a}^{i} \mathrm{~d}^{3} x=0=\int_{\mathcal{V}} \delta K_{a}^{i} \delta_{i}^{a} \mathrm{~d}^{3} x
$$

on the perturbation fields. With these conditions, we obtain the basic Poisson brackets

$$
\{k, p\}=\frac{8 \pi G}{3 V_{0}} \quad, \quad\left\{\delta K_{a}^{i}(x), \delta E_{j}^{b}(y)\right\}=\delta_{a}^{b} \delta_{j}^{i}\left(\delta(x, y)-\frac{1}{V_{0}}\right) .
$$

(The subtraction of the constant $1 / V_{0}$ refers to the Dirac bracket of fields subject to linear second-class constraints, but it will not contribute to the following calculations.)

For a spatially flat isotropic model in triad form, we have the background constraint

$$
\bar{H}=-\frac{3 V_{0}}{8 \pi G} \sqrt{p} k^{2},
$$

the first-order constraint

$$
H^{(1)}[\delta N]=\frac{1}{16 \pi G} \int \mathrm{d}^{3} x \delta N\left(-4 k \sqrt{p} \delta_{j}^{c} \delta K_{c}^{j}-\frac{k^{2}}{\sqrt{p}} \delta_{c}^{j} \delta E_{j}^{c}+\frac{2}{\sqrt{p}} \partial_{c} \partial^{j} \delta E_{j}^{c}\right),
$$

and the second-order constraint

$$
\begin{aligned}
H^{(2)}[\bar{N}]= & \frac{\bar{N}}{16 \pi G} \int \mathrm{d}^{3} x\left(\sqrt{p} \delta K_{c}^{j} \delta K_{d}^{k} \delta_{k}^{d} \delta_{j}^{d}-\sqrt{p}\left(\delta K_{c}^{j} \delta_{j}^{c}\right)^{2}-2 \frac{k}{\sqrt{p}} \delta E_{j}^{c} \delta K_{c}^{j}\right. \\
& \left.-\frac{k^{2}}{2 p^{3 / 2}} \delta E_{j}^{c} \delta E_{k}^{d} \delta_{c}^{k} \delta_{d}^{j}+\frac{k^{2}}{4 p^{3 / 2}}\left(\delta E_{j}^{c} \delta_{j}^{c}\right)^{2}-\frac{1}{2 p^{3 / 2}} \delta^{j k}\left(\partial_{c} \delta E_{j}^{c}\right)\left(\partial_{d} \delta E_{k}^{d}\right)\right) .
\end{aligned}
$$


Moreover, the first-order diffeomorphism constraint is

$$
D\left[\delta M^{c}\right]=\frac{1}{8 \pi G} \int_{\mathcal{V}} \mathrm{d}^{3} x \delta M^{c}\left(p \delta_{k}^{d} \partial_{c} \delta K_{d}^{k}-p \partial_{j} \delta K_{c}^{j}-k \delta_{c}^{j} \partial_{d} \delta E_{j}^{d}\right) .
$$

The background diffeomorphism constraint vanishes identically, and no second-order expression is required for our purposes.

Let us first consider only the background constraint, $\bar{N} \bar{H}$, and the first-order constraint, $H^{(1)}[\delta N]$, in the Poisson bracket

$$
\left\{\bar{N} \bar{H}, H^{(1)}[\delta N]\right\}=\frac{1}{16 \pi G} \int_{\mathcal{V}} \mathrm{d}^{3} x \bar{N} \delta N\left(2 k^{2} \delta_{j}^{c} \delta K_{c}^{j}-2 \frac{k^{3}}{p} \delta_{c}^{j} \delta E_{j}^{c}+2 \frac{k}{p} \partial_{c} \partial^{j} \delta E_{j}^{c}\right) .
$$

It is easy to see that this bracket, which is a first-order expression, is not a linear combination of the available first-order constraints, $H^{(1)}[\delta N]$ and $D\left[\delta M^{c}\right]$. Therefore, if we combine only background and first-order constraints, we not only fail to produce the correct bracket (16) of perturbative hypersurface deformations but, worse, obtain an anomalous gauge system in which the constraint brackets do not close.

This problem can easily be solved by realizing that the second-order constraint $H^{(2)}[\bar{N}]$, while it can be ignored in the constraint equations imposed on first-order dynamics, should be included in the constraint brackets because its Poisson bracket with a first-order constraint is of first order. The second-order constraint therefore contributes to the first-order gauge flow relevant for a theory of first-order perturbations. Indeed, the Poisson bracket

$$
\begin{aligned}
\left\{H^{(2)}[\bar{N}], H^{(1)}[\delta N]\right\}= & \frac{1}{32 \pi G} \int_{\mathcal{V}} \mathrm{d}^{3} x \bar{N}(\delta N( \\
+ & -8 k^{2} \delta_{j}^{c} \delta K_{c}^{j}+4 \frac{k^{3}}{p} \delta_{c}^{j} \delta E_{j}^{c} \\
& \left.+4 \frac{k}{p} \partial_{c} \partial^{j} \delta E_{j}^{c}+4 k^{2} \delta_{j}^{c} \delta K_{c}^{j}\right) \\
& \left.+4\left(\delta K_{c}^{j} \partial_{j} \partial^{c} \delta N-\delta_{j}^{c} \delta K_{c}^{j} \delta_{k}^{d} \partial_{d} \partial^{k} \delta N-\frac{k}{p} \delta E_{j}^{c} \partial_{c} \partial^{j} \delta N\right)\right)
\end{aligned}
$$

provides just the right terms for (31) and (32) to combine to

$$
\begin{aligned}
\left\{\bar{N} \bar{H}+H^{(2)}[\bar{N}], H^{(1)}[\delta N]\right\} & =\frac{1}{8 \pi G} \int_{\mathcal{V}} \mathrm{d}^{3} x \frac{\bar{N} \partial^{c} \delta N}{p}\left(p \delta_{k}^{d} \partial_{c} \delta K_{d}^{j}-p \partial_{j} \delta K_{c}^{j}-k \delta_{c}^{j} \partial_{d} \delta E_{j}^{d}\right) \\
& =D\left[p^{-1} \bar{N} \partial^{c} \delta N\right],
\end{aligned}
$$

equivalent to (16).

With hindsight, the result of this rather technical calculation is not surprising if one only considers that a second-order constraint can generate a first-order gauge flow. Together with the general condition that all flows of the same order should be included on the same footing, it is clear that one cannot obtain an anomaly-free constrained system to first order if only the background and first-order constraints are included. In our following discussion it will be useful to see the presented details of how this calculation works in order to rule out the specific proposal made in the dressed-metric approach. 


\section{The metric's new clothes}

In Riemannian geometry, the metric $g_{\alpha \beta}$ is subject to the tensor transformation law such that the line element

$$
\mathrm{d} s^{2}=g_{\alpha \beta} \mathrm{d} x^{\alpha} \mathrm{d} x^{\beta}
$$

is invariant with respect to coordinate changes, $\mathrm{d} x^{\alpha^{\prime}}=\left(\partial x^{\alpha^{\prime}} / \partial x^{\alpha}\right) \mathrm{d} x^{\alpha}$. The line element therefore provides a coordinate-independent meaning of distances on which Riemannian geometry is based. In a geometrical field theory such as general relativity, this important condition on the metric is an off-shell property which cannot be tested if one restricts one's attention only to solutions of the canonical constraints or to Dirac or other observables.

If the theory is quantized canonically, coordinate transformations are unmodified because the space-time coordinates $x^{\alpha}$ are not phase-space functions. (We ignore here the possibility that one might wish to modify the geometry in addition to canonically quantizing gravity, for instance by making it non-commutative. Such a procedure would go beyond standard canonical quantization, and it is certainly not envisioned in [1].) Some of the components of $g_{\alpha \beta}$, however, represent phase-space degrees of freedom and may therefore be subject to quantum corrections not only in their dynamics but also in their behavior under gauge transformations. The covariance question in canonical quantizations of gravity therefore asks whether a quantum modified (or dressed) $\tilde{g}_{\alpha \beta}$ has off-shell transformations consistent with coordinate transformations. If this question is not answered in the affirmative, the standard interpretation of the metric through a line element is no longer available, demoting $\tilde{g}_{\alpha \beta}$ to a purely formal object without geometrical significance.

In [1], different versions of line elements have uncritically been introduced for modified metrics without asking the covariance question. In fact, since the formalism defined in [1] is purely on-shell, using deparameterization of the background dynamics together with Bardeen potentials or curvature perturbations, it is not amenable to a direct test of covariance. This lack of control on an important physical requirement may in itself present a good reason to discard the dressed metric.

It is possible to go even further and show that the modified dynamics used by the dressed-metric approach in order to obtain bouncing background solutions cannot represent on-shell solutions of a covariant off-shell theory. To do so, we use the canonical version of the tensor-transformation law dual to standard coordinate transformations, given by gauge generators subject to hypersurface-deformation brackets. As we have already seen, perturbative inhomogeneity to first order requires us to use the Hamiltonian constraint up to second order because a second-order contribution may well generate a first-order flow. The dressed metric approach is half-way aware of this important fact because it derives a dynamical flow using second-order generators, determining the dynamical vector field

$$
X_{\text {Dyn }}^{\alpha}=\Omega_{o}^{\alpha \beta} \partial_{\beta} S_{o}\left[N_{\text {hom }}\right]+\Omega_{1}^{\alpha \beta} \partial_{\beta} S_{2}^{\prime}\left[N_{\text {hom }}\right]
$$

in the notation of [1]. The generator $S_{2}^{\prime}$ corresponds to our $H^{(2)}$, but is written in terms 
of curvature perturbations, $T_{\vec{k}}$ and their momenta $P_{\vec{k}}$, for tensor modes:

$$
S_{2}^{\prime}\left[a^{3} \ell^{3} / p_{\phi}\right]=\frac{1}{2} \sum_{\vec{k}}\left(4 \frac{\kappa}{p_{\phi}}\left|P_{\vec{k}}\right|^{2}+\frac{k^{2}}{4 \kappa} \frac{a^{4}}{p_{\phi}}\left|T_{\vec{k}}\right|^{2}\right)
$$

using the choice of lapse function, $N_{\text {hom }}=a^{3} \ell^{3} / p_{\phi}$, preferred in [1]. Here, $p_{\phi}$ is the constant background momentum of the free, massless scalar field used for deparameterization, while $\kappa=8 \pi G$ and $\ell$ is a length parameter that is not relevant for our purposes.

Quantization is then performed separately for $S_{o}$ and $S_{2}^{\prime}$. The background generator $S_{o}$, or our $\bar{H}$, is modified by loop quantization, replacing its quadratic momentum dependence in (27) with a bounded function. (The precise form of this modification does not matter for the arguments given below.) The perturbation part $S_{2}^{\prime}$, however, remains quadratic in momenta and has only slightly modified coefficients,

$$
\tilde{S}_{2}^{\prime}\left[a^{3} \ell^{3} / p_{\phi}\right]=\frac{1}{2} \sum_{\vec{k}}\left(4 \kappa\left\langle\hat{p}_{\phi}^{-1}\right\rangle\left|P_{\vec{k}}\right|^{2}+\frac{k^{2}}{4 \kappa}\left\langle\hat{p}_{\phi}^{-1 / 2} \hat{a}^{4} \hat{p}_{\phi}^{-1 / 2}\right\rangle\left|T_{\vec{k}}\right|^{2}\right)
$$

where background operators are reduced to (internal) time-dependent functions by taking expectation values in a background state. The same expectation values are then used to define a dressed metric in the proposed line element

$$
\mathrm{d} \tilde{s}^{2}=\tilde{g}_{a b} \mathrm{~d} x^{a} \mathrm{~d} x^{b}=-\ell^{6}\left\langle\hat{p}_{\phi}^{-1}\right\rangle^{1 / 2}\left\langle\hat{p}_{\phi}^{-1 / 2} \hat{a}^{4} \hat{p}_{\phi}^{-1 / 2}\right\rangle^{3 / 2} \mathrm{~d} \phi^{2}+\left\langle\hat{p}_{\phi}^{-1}\right\rangle^{-1 / 2}\left\langle\hat{p}_{\phi}^{-1 / 2} \hat{a}^{4} \hat{p}_{\phi}^{-1 / 2}\right\rangle^{1 / 2} \mathrm{~d} \vec{x}^{2},
$$

such that the coefficients in (37) correspond to the classical expression if one were to use the dressed metric to compute it. (The proposal in [1] also includes a metric operator such that

$$
\mathrm{d} \hat{s}^{2}=\hat{g}_{a b} \mathrm{~d} x^{a} \mathrm{~d} x^{b}=-\ell^{6} \hat{p}_{\phi}^{-1} \hat{a}^{6} \hat{p}_{\phi}^{-1} \mathrm{~d} \phi^{2}+\hat{a}^{2} \mathrm{~d} \vec{x}^{2} .
$$

However, since geometrical procedures do not measure operators, this object does not have any well-defined meaning, other than that it produces (38) as a formal expectation value.)

The coefficients of the dressed metric are background functions and are therefore modified if one inserts solutions of the holonomy-modified background constraint. Moreover, there are state-dependent quantum corrections in these coefficients, defined through expectation values, which could be derived systematically in a moment expansion in the framework of effective canonical constraints; see for instance [28, 29, 30]. However, these two quantum corrections cannot counter modifications of the background constraint so as to make the bracket (16) work out, for the following reasons:

- The off-shell behavior of the metric does not depend on what kind of background solutions are entered, and therefore it does not know about holonomy modifications. For the off-shell behavior, relevant for covariance, coefficients in (38) depending on $a$ and $p_{\phi}$ (and possibly their moments) are merely phase-space coordinates, just like the corresponding functions in the modified background constraint. The off-shell theory of the dressed-metric approach therefore corresponds to a system in which 
only the background constraint, $\bar{H}$, has been modified by using holonomies, but not the second-order constraint, $H^{(2)}$. Moreover, also the first-order constraint, $H^{(1)}$ is unmodified because [1] uses the classical curvature perturbations without modifications that would result if gauge transformations generated by $H^{(1)}$ were modified; see [26, 31]. The bracket (32) then remains unchanged while (31) is modified, eliminating important cancellations that led to the combined result (33). The dressed-metric approach functions by modifying only the background constraint, making it impossible to realize a valid version of the perturbative hypersurface-deformation bracket (16).

- If moments of a state that result from a systematic semiclassical expansion of the expectation values in (37) were to counter the background modification, they would have to be fixed, severely restricting the class of quantum states that are allowed to propagate. Even if there were moments such that the bracket (16) could be closed after background modifications, the resulting mismatch of classical and quantum degrees of freedom would amount to an anomaly. (Recall that an anomaly in a constraint system implies that the system becomes over-constrained, imposing an additional constraint such as $\left\{\bar{N} \bar{H}, H^{(1)}[\delta N]\right\}=0$ if the left-hand side is no longer zero on the solutions space of the original constraints.)

In addition to violating covariance, the dressed metric has the following problem: The dressed metric depends on the ordering chosen for operators in the expectation-value components. Moreover, for different background gauges, corresponding to different phase-space function for the background lapse $\bar{N}$, different operator products appear, giving rise to different ordering ambiguities. Therefore, choosing a different background gauge in general results in an inequivalent dressed metric. Ordering issues can potentially be ignored if one uses sharply peaked states, such that fluctuation terms are negligible. Such an assumption is sometimes suggested by the dressed-metric approach, as in "one knowns that there exist background quantum geometries $\Psi_{o}$ which are very sharply peaked" (emphasis in [1]). However, this assumption is not justified in the Planck regime [32, 33], where a dressed metric would be most relevant.

\section{Conclusions}

Covariance in canonical quantum gravity is a subtle issue. It requires a formulation of quantum effects such that the classical hypersurface-deformation brackets (15) are obtained in the classical limit of the theory, while a closed, anomaly-free set of brackets is realized for non-zero $\hbar$ which vanishes when the constraints are solved but is not necessarily of the classical form. This statement includes two conditions, which cannot always both be met. For instance, a possible Abelianization of the bracket in some midisuperspace models [34, 35] always leads to anomaly-free quantum constraints but even then is not guaranteed to be compatible with covariance [36, 37]. Although such quantum theories in

the latter case are formally consistent as quantizations of constrained systems, they cannot 
be interpreted as models of quantum space-time because there is no well-defined sense in which they are covariant.

As an alternative to realizations of the hypersurface-deformation brackets, analog actions in space-time tensor form, such as certain scalar-tensor theories, have been proposed as a possible way to demonstrate covariance. However, while such analog actions may work in simple, isotropic models with a small number of degrees of freedom, in all known cases they fail to describe anisotropic models or perturbative inhomogeneity correctly. For instance, the Palatini- $f(R)$ model proposed in [38], claimed to show that loop quantum cosmology is covariant, is equivalent to a scalar-tensor theory with a non-dynamical scalar [39] in which any correction to general relativity amounts to a simple cosmological constant in vacuum models. It therefore cannot possibly describe holonomy modifications in anisotropic vaccum models, ruling it out as a possible covariant version of loop quantum cosmology. More recent analog actions [40, 41] based on mimetic gravity [42] again work in isotropic models but fail to describe anisotropies or perturbative inhomogeneity correctly [43, 44, 45].

As shown here, the dressed-metric approach again fails to provide a covariant version of perturbative inhomogeneity in loop quantum cosmology, in particular in the presence of holonomy modifications of the background dynamics that may make it possible to have bouncing solutions. Although we have focused on the specific formulation described in 1 . for technical details of the constructions, similar arguments apply to related (or precursor) formulations in [46, 47] or the "hybrid" approach [48, 49, 50] which share with the dressedmetric approach the crucial feature of separating the background degrees of freedom from inhomogeneous modes, making it impossible to implement the key relation (16) which belongs to a semidirect product of Lie algebroids.

Our result adds to mounting evidence that models of loop quantum gravity cannot be covariant without drastic modifications of space-time structure; see also [51]. It is sometimes suggested that a non-covariant model which implements some quantum effects in an otherwise consistent way may be useful as a "first approximation" to a complicated formulation of cosmological dynamics in full quantum gravity. However, violating an important consistency condition such as covariance is not an approximation at all because it usually gives rise to uncontrolled, spurious solutions that overshadow the relevant behavior, or to over-constrained dynamics. (See also [52] for a similar result in a different setting.) As an example, covariant versions of holonomy-modified models of loop quantum gravity, derived in [53, 31, 54, 55, 56, generically imply signature change at Planckian density. The wouldbe bounce is then a 4-dimensional Euclidean region in which no deterministic evolution exists [57, 58]. Non-deterministic behavior is an example for an effect that cannot be considered a small correction to modified but still deterministic dynamics, even if the modes used to determine the structure of space-time and propagation properties are perturbative. 


\section{Acknowledgements}

This work was supported in part by NSF grant PHY-1912168. The author thanks Jakub Mielczarek for discussions.

\section{References}

[1] I. Agulló, A. Ashtekar, and W. Nelson, An Extension of the Quantum Theory of Cosmological Perturbations to the Planck Era, Phys. Rev. D 87 (2013) 043507, arXiv:1211.1354

[2] M. Bojowald, Loop Quantum Cosmology, Living Rev. Relativity 11 (2008) 4, |gr-qc/0601085, http://www. livingreviews.org/lrr-2008-4

[3] J. M. Bardeen, Gauge-invariant cosmological perturbations, Phys. Rev. D 22 (1980) $1882-1905$

[4] V. F. Mukhanov, H. A. Feldman, and R. H. Brandenberger, Theory of cosmological perturbations, Phys. Rept. 215 (1992) 203-333

[5] P. A. M. Dirac, Generalized Hamiltonian dynamics, Can. J. Math. 2 (1950) 129-148

[6] W. F. Blyth and C. J. Isham, Quantization of a Friedmann universe filled with a scalar field, Phys. Rev. D 11 (1975) 768-778

[7] P. Malkiewicz, Reduced phase space approach to Kasner universe and the problem of time in quantum theory, Class. Quantum Grav. 29 (2012) 075008, arXiv:1105.6030.

[8] P. Malkiewicz, Multiple choices of time in quantum cosmology, Class. Quantum Grav. 32 (2015) 135004, arXiv:1407.3457]

[9] M. Bojowald and T. Halnon, Time in quantum cosmology, Phys. Rev. D 98 (2018) 066001, arXiv:1612.00353.

[10] M. Bojowald, P. A. Höhn, and A. Tsobanjan, An effective approach to the problem of time, Class. Quantum Grav. 28 (2011) 035006, arXiv:1009.5953.

[11] M. Bojowald, P. A. Höhn, and A. Tsobanjan, An effective approach to the problem of time: general features and examples, Phys. Rev. D 83 (2011) 125023, arXiv:1011.3040.

[12] P. A. Höhn, E. Kubalova, and A. Tsobanjan, Effective relational dynamics of a nonintegrable cosmological model, Phys. Rev. D 86 (2012) 065014, arXiv:1111.5193]

[13] F. Giacomini, A. Castro-Ruiz, and C. Brukner, Quantum mechanics and the covariance of physical laws in quantum reference frames, Nat. Commun. 10 (2019) 494, arXiv:1712.07207 
[14] A. Vanrietvelde, P. A. Hoehn, F. Giacomini, and E. Castro-Ruiz, A change of perspective: switching quantum reference frames via a perspective-neutral framework, arXiv:1809.00556]

[15] A. Vanrietvelde, P. A. Hoehn, and F. Giacomini, Switching quantum reference frames in the $N$-body problem and the absence of global relational perspectives, arXiv:1809.05093

[16] P. A. Hoehn, A. R. H. Smith, and M. P. E. Lock, The Trinity of Relational Quantum Dynamics, arXiv:1912.00033

[17] J. M. Stewart, Perturbations of Friedmann-Robertson-Walker cosmological models, Class. Quantum Grav. 7 (1990) 1169-1180

[18] J. M. Pons, D. C. Salisbury, and L. C. Shepley, Gauge transformations in the Lagrangian and Hamiltonian formalisms of generally covariant theories, Phys. Rev. D 55 (1997) 658-668, gr-qc/9612037

[19] M. Bojowald, Canonical Gravity and Applications: Cosmology, Black Holes, and Quantum Gravity, Cambridge University Press, Cambridge, 2010

[20] C. Blohmann, M. C. Barbosa Fernandes, and A. Weinstein, Groupoid symmetry and constraints in general relativity. 1: kinematics, Commun. Contemp. Math. 15 (2013) 1250061, arXiv:1003.2857]

[21] J. M. Glowacki, Groupoid symmetry, constraints and quantization of General Relativity, arXiv:1905.03152

[22] R. Arnowitt, S. Deser, and C. W. Misner, The Dynamics of General Relativity, In L. Witten, editor, Gravitation: An Introduction to Current Research, Wiley, New York, 1962, Reprinted in [59]

[23] P. A. M. Dirac, The theory of gravitation in Hamiltonian form, Proc. Roy. Soc. A 246 (1958) 333-343

[24] E. Aldrovandi, U. Bruzzo, and V. Rubtsov, Lie algebroid cohomology and Lie algebroid extensions, J. Algebra 505 (2018) 456-481, arXiv:1711.05156]

[25] M. Bojowald, G. Hossain, M. Kagan, and S. Shankaranarayanan, Anomaly freedom in perturbative loop quantum gravity, Phys. Rev. D 78 (2008) 063547, arXiv:0806.3929

[26] M. Bojowald, G. Hossain, M. Kagan, and S. Shankaranarayanan, Gauge invariant cosmological perturbation equations with corrections from loop quantum gravity, Phys. Rev. D 79 (2009) 043505, arXiv:0811.1572.

[27] D. Langlois, Hamiltonian formalism and gauge invariance for linear perturbations in inflation, Class. Quant. Grav. 11 (1994) 389-407 
[28] M. Bojowald and A. Skirzewski, Effective Equations of Motion for Quantum Systems, Rev. Math. Phys. 18 (2006) 713-745, math-ph/0511043

[29] M. Bojowald, B. Sandhöfer, A. Skirzewski, and A. Tsobanjan, Effective constraints for quantum systems, Rev. Math. Phys. 21 (2009) 111-154, arXiv:0804.3365]

[30] M. Bojowald and A. Tsobanjan, Effective constraints for relativistic quantum systems, Phys. Rev. D 80 (2009) 125008, arXiv:0906.1772]

[31] T. Cailleteau, L. Linsefors, and A. Barrau, Anomaly-free perturbations with inversevolume and holonomy corrections in Loop Quantum Cosmology, Class. Quantum Grav. 31 (2014) 125011, arXiv:1307.5238

[32] M. Bojowald, The BKL scenario, infrared renormalization, and quantum cosmology, JCAP 01 (2019) 026, arXiv:1810.00238.

[33] M. Bojowald, Effective field theory of loop quantum cosmology, Universe 5 (2019) 44, arXiv:1906.01501]

[34] R. Gambini and J. Pullin, Loop quantization of the Schwarzschild black hole, Phys. Rev. Lett. 110 (2013) 211301, arXiv:1302.5265]

[35] R. Gambini, J. Olmedo, and J. Pullin, Quantum black holes in Loop Quantum Gravity, arXiv:1310.5996

[36] M. Bojowald, S. Brahma, and J. D. Reyes, Covariance in models of loop quantum gravity: Spherical symmetry, Phys. Rev. D 92 (2015) 045043, arXiv:1507.00329]

[37] M. Bojowald and S. Brahma, Covariance in models of loop quantum gravity: Gowdy systems, Phys. Rev. D 92 (2015) 065002, arXiv:1507.00679]

[38] G. J. Olmo and P. Singh, Covariant Effective Action for Loop Quantum Cosmology a la Palatini, JCAP 0901 (2009) 030, arXiv:0806.2783

[39] G. J. Olmo, The gravity lagrangian according to solar system experiments, Phys. Rev. Lett. 95 (2005) 261102, [gr-qc/0505101]

[40] N. Bodendorfer, A. Schäfer, and J. Schliemann, On the canonical structure of general relativity with a limiting curvature and its relation to loop quantum gravity, Phys. Rev. D 97 (2018) 084057, arXiv:1703.10670.

[41] D. Langlois, H. Liu, K. Noui, and E. Wilson-Ewing, Effective loop quantum cosmology as a higher-derivative scalar-tensor theory, Class. Quant. Grav. 34 (2017) 225004, arXiv:1703.10812

[42] A. H. Chamseddine and V. Mukhanov, Mimetic Dark Matter, JHEP 11 (2013) 135, arXiv:1308.5410 
[43] N. Bodendorfer, F. M. Mele, and J. Münch, Is limiting curvature mimetic gravity an effective polymer quantum gravity?, Class. Quantum Grav. 35 (2018) 225001, arXiv:1806.02052

[44] J. Haro, Ll. Aresté Saló, and S. Pan, Mimetic Loop Quantum Cosmology, Gen. Rel. Grav. 51 (2019) 49, arXiv:1803.09653

[45] J. Ben Achour, F. Lamy, H. Liu, and K. Noui, Non-singular black holes and the limiting curvature mechanism: a Hamiltonian perspective, JCAP 05 (2018) 072, arXiv:1712.03876

[46] A. Ashtekar, W. Kaminski, and J. Lewandowski, Quantum field theory on a cosmological, quantum space-time, Phys. Rev. D 79 (2009) 064030, arXiv:0901.0933]

[47] A. Dapor, J. Lewandowski, and J. Puchta, QFT on quantum spacetime: a compatible classical framework, Phys. Rev. D 87 (2013) 104038, arXiv:1302.3038

[48] M. Martín-Benito, L. J. Garay, and G. A. Mena Marugán, Hybrid Quantum Gowdy Cosmology: Combining Loop and Fock Quantizations, Phys. Rev. D 78 (2008) 083516, arXiv:0804.1098

[49] L. J. Garay, M. Martín-Benito, and G. A. Mena Marugán, Inhomogeneous Loop Quantum Cosmology: Hybrid Quantization of the Gowdy Model, Phys. Rev. D 82 (2010) 044048, arXiv:1005.5654]

[50] M. Fernández-Méndez, G. A. Mena Marugán, and J. Olmedo, Hybrid quantization of an inflationary model: The flat case, Phys. Rev. D 88 (2013) 044013, arXiv:1307.5222

[51] M. Bojowald, Comment (2) on "Quantum Transfiguration of Kruskal Black Holes", arXiv:1906.04650

[52] S. Shankaranarayanan and M. Lubo, Gauge-invariant perturbation theory for transPlanckian inflation, Phys. Rev. D 72 (2005) 123513, hep-th/0507086

[53] J. D. Reyes, Spherically Symmetric Loop Quantum Gravity: Connections to 2Dimensional Models and Applications to Gravitational Collapse, PhD thesis, The Pennsylvania State University, 2009

[54] M. Bojowald, J. D. Reyes, and R. Tibrewala, Non-marginal LTB-like models with inverse triad corrections from loop quantum gravity, Phys. Rev. D 80 (2009) 084002, arXiv:0906.4767

[55] M. Bojowald, G. M. Paily, and J. D. Reyes, Discreteness corrections and higher spatial derivatives in effective canonical quantum gravity, Phys. Rev. D 90 (2014) 025025, arXiv:1402.5130 
[56] S. Brahma, Spherically symmetric canonical quantum gravity, Phys. Rev. D 91 (2015) 124003, arXiv:1411.3661

[57] M. Bojowald and J. Mielczarek, Some implications of signature-change in cosmological models of loop quantum gravity, JCAP 08 (2015) 052, arXiv:1503.09154]

[58] M. Bojowald, Information loss, made worse by quantum gravity, Front. Phys. 3 (2015) 33, arXiv:1409.3157

[59] R. Arnowitt, S. Deser, and C. W. Misner, The Dynamics of General Relativity, Gen. Rel. Grav. 40 (2008) 1997-2027 